\title{
The Contour Integral Method for Loaded Cracks
}

\author{
J. P. Pereira and C.A. Duarte \\ Department of Civil and Environmental Engr., University of Illinois at Urbana-Champaign, \\ Newmark Laboratory, 205 North Mathews Avenue, Urbana, Illinois 61801, USA \\ e-mails: jpereir2@uiuc.edu, caduarte@uiuc.edu
}

\begin{abstract}
An extension of the contour integral method (CIM) for the computation of stress intensity factors for loaded cracks is presented. Numerical solutions of the two-dimensional linear elasticity equations are computed with the $p$-version of the generalized finite element method. Polynomial enrichment functions as well as enrichment with near crack tip asymptotic expansion are used. The robustness of the formulation with respect to the size of the extraction domain and the convergence of the computed stress intensity factors and energy release rate are analyzed.
\end{abstract}

keywords: Stress intensity factors, loaded cracks, contour integral method, generalized finite element method.

\section{Introduction}

Crack behavior analysis involves the evaluation of parameters such as the energy release rate and stress intensity factors (SIF). Several techniques have been proposed to extract these quantities using numerical methods like the finite element and boundary element methods. Several of the extraction methods are based on the J-integral [12] or on one of its variations like the domain equivalent integral $[5,8]$. The interaction energy integral method $[8]$ is a popular technique for the extraction of mixedmode stress intensity factors. It is derived from the J-integral by considering auxiliary fields. A review of methods for calculating energy release rates can be found in [5].

The Contour Integral Method (CIM) is a superconvergent technique for the extraction of stress intensity factors proposed by Szabo and Babuska [16] within the framework of the $p$-version of the finite element method. This method is based on the computation of functionals from numerical solutions and the so-called extraction functions. It is a superconvergent technique since the computed quantities converge to their true values at least as fast as the strain energy. This method can be used to compute the amplitudes of any number of terms of the asymptotic expansion of the elasticity solution at reentrant corners. In particular, it can be used to compute stress intensity factors from mixed mode crack problems.

In the analysis of cracks in structures like thick walled pressured vessels, it is necessary for realistic predictions to account for the internal pressure on the crack faces [1]. The analysis of cohesive crack growth is another example in which crack faces are loaded. In this paper, the CIM is generalized for the case of cracks with tractions applied to their faces. The formulation presented by Szabo and Babuska [16] is shown to be a special case of the one presented here. Numerical examples demonstrate that, like in the case of stress-free crack faces, computed stress intensity factors converge exponentially. 
The $p$-version of the generalized finite element method $[2,9,14]$ is used to compute approximate solutions of the elasticity equations. Polynomial enrichment functions as well as enrichment with Westergaard near crack tip expansion is investigated.

The cutoff function method (CFM) [16] is an extraction technique closely related to the CIM. The extension of the CFM to the case of pressurized cracks is presented in [10].

The paper is organized as follows. After this introduction, an integral identity and the so-called extraction functions are presented in Section 2. The formulation of the contour integral method for cracks with tractions applied to their faces is presented in Section 2.1. A short summary of the generalized finite element method is presented in Section 3. A Numerical example is presented next and the main conclusions of this work are drawn in Section 5.

\section{Extraction of Stress Intensity Factors}

Consider a cracked plane elastic domain $\Omega$ and coordinate systems $(x, y)$ and $\left(x_{1}, x_{2}\right)$ as illustrated in Fig. 1. Let

$$
\mathbf{u}\left(x_{1}, x_{2}\right)=\left\{\begin{array}{l}
u_{1}\left(x_{1}, x_{2}\right) \\
u_{2}\left(x_{1}, x_{2}\right)
\end{array}\right\}
$$

denote the displacement field in $\Omega$ written in terms of the local Cartesian coordinates $\left(x_{1}, x_{2}\right)$ associated with the crack tip.

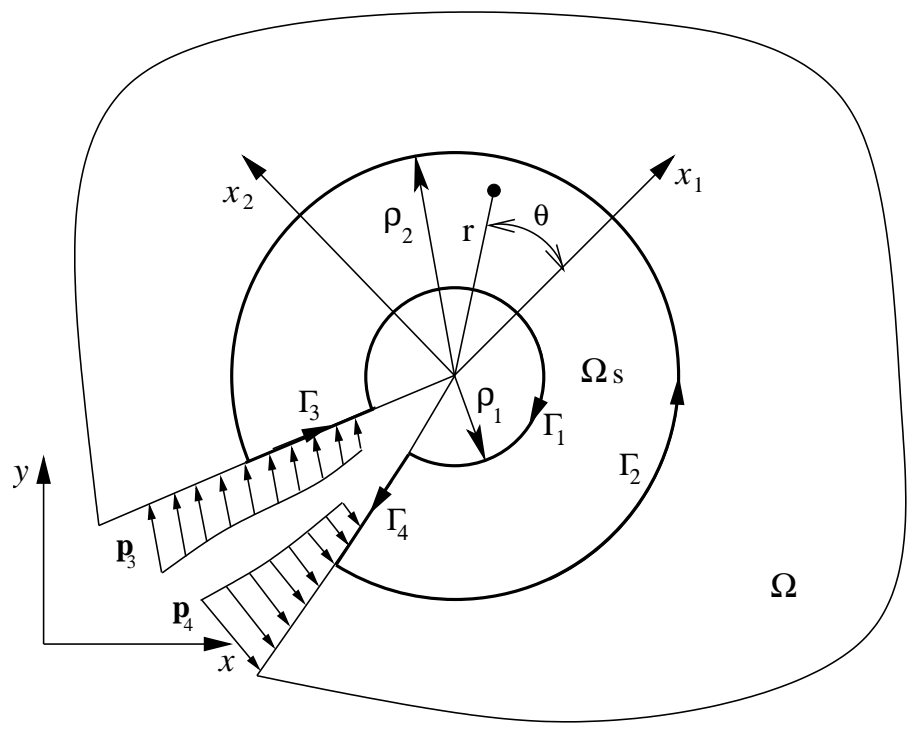

Figure 1: Coordinated systems, domain $\Omega$ and neighborhood $\Omega_{s}$ around the crack tip. The crack opening is shown for illustration only. The $x_{1}$-axis is parallel to the crack faces.

The two dimensional linear elasticity equilibrium equations on a domain $\Omega$, in the absence of body forces, are given by

$$
\sigma_{i j, j}^{(\mathbf{u})}=0 \quad \text { in } \Omega
$$

where

$$
\begin{aligned}
\sigma_{i j}^{(\mathbf{u})} & =D_{i j k l} \varepsilon_{k l}^{(\mathbf{u})} \\
\varepsilon_{i j}^{(\mathbf{u})} & =\frac{1}{2}\left(u_{i, j}+u_{j, i}\right)
\end{aligned}
$$


with $D_{i j k l}$ being the tensor of elastic constants for a homogeneous isotropic material with Young's modulus $E$ and Poisson's ratio $\nu$. For two dimensional case,

$$
\begin{aligned}
& D_{1111}=D_{2222}=A \\
& D_{1122}=D_{2211}=\nu A \\
& D_{1212}=E /(1+\nu)
\end{aligned}
$$

where

$$
A= \begin{cases}E /\left(1-\nu^{2}\right) & \text { for plane stress } \\ E(1-\nu) /[(1+\nu)(1-2 \nu)] & \text { for plane strain }\end{cases}
$$

and all other components of $D$ are zero. The direction cosines of the normal to the boundary of the body $\Omega$ at a point $P$ are denoted by $\left(n_{1}, n_{2}\right)$. The traction vector components at a point $P \in \partial \Omega$ are given by

$$
T_{i}^{(\mathbf{u})}=\sigma_{i j}^{(\mathbf{u})} n_{j} \quad \text { on } \partial \Omega
$$

where $\sigma_{i j}^{(\mathbf{u})}$ and $T_{i}^{(\mathbf{u})}$ denote stress components and the components of the traction vector on the boundary $\partial \Omega$, respectively, computed from the displacement field $\mathbf{u}$.

Herein we assume that the crack faces, $\Gamma_{3}$ and $\Gamma_{4}$, are loaded by prescribed tractions given by

$$
\boldsymbol{T}^{(\mathbf{u})}=\left\{\begin{array}{lll}
\mathbf{p}^{3} & \text { on } & \Gamma_{3} \\
\mathbf{p}^{4} & \text { on } & \Gamma_{4}
\end{array}\right.
$$

Appropriate boundary conditions are applied on $\partial \Omega \backslash\left(\Gamma_{3} \cup \Gamma_{4}\right)$.

Let

$$
\mathbf{v}\left(x_{1}, x_{2}\right)=\left\{\begin{array}{l}
v_{1}\left(x_{1}, x_{2}\right) \\
v_{2}\left(x_{1}, x_{2}\right)
\end{array}\right\}
$$

denote a virtual displacement field defined on $\Omega$.

Consider now a subdomain $\Omega_{s}$ in the neighborhood of the crack tip with boundary $\partial \Omega_{s}=\Gamma_{1} \cup$ $\Gamma_{2} \cup \Gamma_{3} \cup \Gamma_{4}$ and internal (external) radius $\rho_{1}\left(\rho_{2}\right)$ as illustrated in Fig. 1. Using the definitions above and following the standard derivation of the Principle of Virtual Work (e.g. [17]) it can be shown that

$$
\int_{\Omega_{s}} \sigma_{i j}^{(\mathbf{u})} \varepsilon_{i j}^{(\mathbf{v})} d \Omega=\int_{\partial \Omega_{s}} T_{i}^{(\mathbf{u})} v_{i} d \Gamma
$$

holds for any virtual displacement field $\mathbf{v}$ with an associated finite strain energy in $\Omega_{s}$. In the above, $\varepsilon_{i j}^{(\mathbf{v})}$ denotes the strain components computed from the virtual displacement field $\mathbf{v}$.

If the displacement field $\mathbf{v}$ satisfies the equilibrium equations, the equation above leads, after some standard manipulations, to the so-called Betti's law [16]

$$
\int_{\partial \Omega_{s}} T_{k}^{(\mathbf{v})} u_{k} d \Gamma=\int_{\partial \Omega_{s}} T_{i}^{(\mathbf{u})} v_{i} d \Gamma
$$

The integral equation above is used in Section 2.1 to derive the contour integral method.

Let

$$
\overline{\mathbf{u}}(r, \theta)=\left\{\begin{array}{l}
\bar{u}_{1}(r, \theta) \\
\bar{u}_{2}(r, \theta)
\end{array}\right\}
$$


denote the displacement field in $\Omega_{s}$ written in terms of the local polar coordinates $(r, \theta)$ associated with the crack tip (Cf. Fig. 1). Herein, bar quantities, "”, , are defined in terms of local polar coordinates.

If the internal radius, $\rho_{1}$, of the subdomain $\Omega_{s}$ is sufficiently small, the displacement field on $\Gamma_{1}$ can be approximated by the first term of the asymptotic near-tip expansion [16]

$$
\begin{aligned}
\overline{\mathbf{u}}(r, \theta)=\left\{\begin{array}{l}
\bar{u}_{1}(r, \theta) \\
\bar{u}_{2}(r, \theta)
\end{array}\right\} & =\frac{K_{I}}{2 G \sqrt{2 \pi}} \sqrt{r}\left\{\begin{array}{c}
\left(\kappa-\frac{1}{2}\right) \cos \frac{\theta}{2}-\frac{1}{2} \cos \frac{3 \theta}{2} \\
\left(\kappa+\frac{1}{2}\right) \sin \frac{\theta}{2}-\frac{1}{2} \sin \frac{3 \theta}{2}
\end{array}\right\} \\
& +\frac{K_{I I}}{2 G \sqrt{2 \pi}} \sqrt{r}\left\{\begin{array}{c}
\left(\kappa+\frac{3}{2}\right) \sin \frac{\theta}{2}+\frac{1}{2} \sin \frac{3 \theta}{2} \\
-\left[\left(\kappa-\frac{3}{2}\right) \cos \frac{\theta}{2}+\frac{1}{2} \cos \frac{3 \theta}{2}\right]
\end{array}\right\}
\end{aligned}
$$

where $K_{I}\left(K_{I I}\right)$ is the mode I (II) stress intensity factor, $G$ is the shear modulus of rigidity and $\kappa$ is the Kolosov's constant

$$
\kappa= \begin{cases}(3-\nu) /(1+\nu) & \text { for plane stress } \\ 3-4 \nu & \text { for plane strain }\end{cases}
$$

The traction vector computed from the displacement field $\overline{\mathbf{u}}(r, \theta)$ is denoted by $\overline{\boldsymbol{T}}^{(\overline{\mathbf{u}})}(r, \theta)$.

Based on the displacement field presented in Eq. (3), the so-called extraction functions for mode I and mode II are defined, respectively, by [17]

$$
\begin{aligned}
& \overline{\mathbf{v}}^{-I}(r, \theta)=\left\{\begin{array}{l}
\bar{v}_{1}^{-I}(r, \theta) \\
\bar{v}_{2}^{-I}(r, \theta)
\end{array}\right\}=\frac{B^{-I}}{2 G \sqrt{2 \pi}} \frac{1}{\sqrt{r}}\left\{\begin{array}{c}
\left(\kappa-\frac{3}{2}\right) \cos \frac{\theta}{2}+\frac{1}{2} \cos \frac{5 \theta}{2} \\
-\left(\kappa+\frac{3}{2}\right) \sin \frac{\theta}{2}+\frac{1}{2} \sin \frac{5 \theta}{2}
\end{array}\right\} \\
& \overline{\mathbf{v}}^{-I I}(r, \theta)=\left\{\begin{array}{l}
\bar{v}_{1}^{-I I}(r, \theta) \\
\bar{v}_{2}^{-I I}(r, \theta)
\end{array}\right\}=\frac{B^{-I I}}{2 G \sqrt{2 \pi}} \frac{1}{\sqrt{r}}\left\{\begin{array}{c}
-\left(\kappa+\frac{1}{2}\right) \sin \frac{\theta}{2}-\frac{1}{2} \sin \frac{5 \theta}{2} \\
-\left[\left(\kappa-\frac{1}{2}\right) \cos \frac{\theta}{2}-\frac{1}{2} \cos \frac{5 \theta}{2}\right]
\end{array}\right\}
\end{aligned}
$$

where $B^{-I}$ and $B^{-I I}$ are constants defined later. Equations (4) and (5) play a key role in the definition of the extraction method discussed here. The extraction function for a given mode is defined by taking the same expression of the asymptotic expansion but using the negative of the eigenvalue associated with the mode. A detailed procedure showing how to define the extraction functions can be found in [17]. These functions do not have finite strain energy in $\Omega$. However, they have finite strain energy in $\Omega_{s}$ and satisfy the equilibrium equations.

The traction vectors computed from $\overline{\mathbf{v}}^{-I}$ and $\overline{\mathbf{v}}^{-I I}$ are denoted by $\overline{\boldsymbol{T}}^{\left(\overline{\mathbf{v}}^{-I}\right)}$ and $\overline{\boldsymbol{T}}^{\left(\overline{\mathbf{v}}^{-I I}\right)}$, respectively. It is not difficult to verify that these tractions are zero on the crack faces, i.e., on $\Gamma_{3}$ and $\Gamma_{4}$.

\subsection{The Contour Integral Method for Loaded Cracks}

In this section, the integral identity (2) is used to derive the contour integral method (CIM) for cracks with tractions applied to their faces. Let us consider first the integral on the left hand side of Eq. (2), i.e.,

$$
\int_{\partial \Omega_{s}} T_{k}^{(\mathbf{v})} u_{k} d \Gamma \equiv \int_{\Gamma_{1} \cup \Gamma_{2} \cup \Gamma_{3} \cup \Gamma_{4}} T_{k}^{(\mathbf{v})} u_{k} d \Gamma=\int_{\Gamma_{1} \cup \Gamma_{2} \cup \Gamma_{3} \cup \Gamma_{4}} \bar{T}_{k}^{(\overline{\mathbf{v}})} \bar{u}_{k} d \Gamma
$$

Let $\overline{\mathbf{v}}$ denote the extraction function $\overline{\mathbf{v}}^{-I}$ or $\overline{\mathbf{v}}^{-I I}$. Then

$$
\int_{\Gamma_{3}} \bar{T}_{k}^{(\overline{\mathbf{v}})} \bar{u}_{k} d \Gamma=\int_{\Gamma_{4}} \bar{T}_{k}^{(\overline{\mathbf{v}})} \bar{u}_{k} d \Gamma=0
$$

since $\overline{\boldsymbol{T}}^{\left(\overline{\mathbf{v}}^{-I}\right)}(r, \theta)$ and $\overline{\boldsymbol{T}}^{\left(\overline{\mathbf{v}}^{-I I}\right)}(r, \theta)$ are zero on the crack faces. 
Let $\overline{\mathbf{v}}=\overline{\mathbf{v}}^{-I}$, then it can be shown that ${ }^{1}$

$$
\int_{\Gamma_{1}} \bar{T}_{k}^{\left(\overline{\mathbf{v}}^{-I}\right)} \bar{u}_{k} d \Gamma=C_{l}^{-I} B^{-I} K_{I}
$$

where

$$
C_{l}^{-I}=\frac{-(3 \kappa+1)}{8 G}
$$

and $\kappa$ is the Kolosov's constant.

Similarly, if $\overline{\mathbf{v}}=\overline{\mathbf{v}}^{-I I}$, then,

$$
\int_{\Gamma_{1}} \bar{T}_{k}^{\left(\overline{\mathbf{v}}^{-I I}\right)} \bar{u}_{k} d \Gamma=C_{l}^{-I I} B^{-I I} K_{I I}
$$

where

$$
C_{l}^{-I I}=\frac{-(\kappa+3)}{8 G}
$$

Let us now consider the integral on the right hand side of Eq. (2), i.e.,

$$
\int_{\partial \Omega_{s}} T_{i}^{(\mathbf{u})} v_{i} d \Gamma \equiv \int_{\Gamma_{1} \cup \Gamma_{2} \cup \Gamma_{3} \cup \Gamma_{4}} T_{i}^{(\mathbf{u})} v_{i} d \Gamma=\int_{\Gamma_{1} \cup \Gamma_{2} \cup \Gamma_{3} \cup \Gamma_{4}} \bar{T}_{i}^{(\overline{\mathbf{u}})} \bar{v}_{i} d \Gamma
$$

Let $\overline{\mathbf{v}}=\overline{\mathbf{v}}^{-I}$, then it can be shown that

$$
\int_{\Gamma_{1}} \bar{T}_{i}^{(\overline{\mathbf{u}})} \bar{v}_{i}^{-I} d \Gamma=C_{r}^{-I} B^{-I} K_{I}
$$

where

$$
C_{r}^{-I}=\frac{(\kappa+3)}{8 G}
$$

Similarly, if $\overline{\mathbf{v}}=\overline{\mathbf{v}}^{-I I}$ then,

$$
\int_{\Gamma_{1}} \bar{T}_{i}^{(\overline{\mathbf{u}})} \bar{v}_{i}^{-I I} d \Gamma=C_{r}^{-I I} B^{-I I} K_{I I}
$$

where

$$
C_{r}^{-I I}=\frac{(3 \kappa+1)}{8 G}
$$

From Eq. (2) and the above we have that

$$
\begin{aligned}
C_{l}^{-I} B^{-I} K_{I}+\int_{\Gamma_{2}} \bar{T}_{k}^{\left(\overline{\mathbf{v}}^{-I}\right)} \bar{u}_{k} d \Gamma & =C_{r}^{-I} B^{-I} K_{I}+\int_{\Gamma_{2}} \bar{T}_{i}^{(\overline{\mathbf{u}})} \bar{v}_{i}^{-I} d \Gamma \\
& +\int_{\Gamma_{3}} \bar{p}_{i}^{3} \bar{v}_{i}^{-I} d \Gamma+\int_{\Gamma_{4}} \bar{p}_{i}^{4} \bar{v}_{i}^{-I} d \Gamma
\end{aligned}
$$

Therefore, adopting $B^{-I}:=1 /\left(C_{l}^{-I}-C_{r}^{-I}\right)=-2 G /(\kappa+1)$, and using local Cartesian coordinates instead of polar coordinates, we have that

$$
K_{I}=\int_{\Gamma_{2}} T_{i}^{(\mathbf{u})} v_{i}^{-I} d \Gamma-\int_{\Gamma_{2}} T_{k}^{\left(\mathbf{v}^{-I}\right)} u_{k} d \Gamma+\int_{\Gamma_{3}} p_{i}^{3} v_{i}^{-I} d \Gamma+\int_{\Gamma_{4}} p_{i}^{4} v_{i}^{-I} d \Gamma
$$

Similarly, $K_{I I}$ can be computed using

$$
K_{I I}=\int_{\Gamma_{2}} T_{i}^{(\mathbf{u})} v_{i}^{-I I} d \Gamma-\int_{\Gamma_{2}} T_{k}^{\left(\mathbf{v}^{-I I}\right)} u_{k} d \Gamma+\int_{\Gamma_{3}} p_{i}^{3} v_{i}^{-I I} d \Gamma+\int_{\Gamma_{4}} p_{i}^{4} v_{i}^{-I I} d \Gamma
$$

It is important to point out that

\footnotetext{
${ }^{1}$ We employ symbolic computation to compute integrals like the one in (6). The Maple code is available by contacting the authors.
} 
- The expressions above for $K_{I}$ and $K_{I I}$ are exact. However, neither the solution u nor the corresponding traction $\mathbf{T}^{(\mathbf{u})}$ are known. In this paper, we use instead numerical solutions provided by the generalized finite element method (Cf. Section 3) to obtain approximations to $K_{I}$ and $K_{I I}$.

- In the above, if $\mathbf{p}^{3}=\mathbf{p}^{4}=\mathbf{0}$ then Eqs. (10) and (11) reduce to the formulation presented in [16].

- The contour $\Gamma_{2}$ can be as large as we wish, in other words, $\Gamma_{2}$ does not have to be close to the crack tip. This property of the CIM is confirmed in the numerical experiments presented in Section 4. This is in contrast, for example, with the J-integral method. This is important since numerical solutions are of lesser quality near the crack tip.

- The path $\Gamma_{2}$ does not have to be circular as used in the derivations above. The choice of its shape is mostly dictated by computational implementation issues. An implementation of the CIM and the CFM that can be used with most numerical methods is proposed in [11].

\section{The Generalized Finite Element Method - A Summary}

The generalized finite element method $[2,6,9,14]$ is used in the next section to compute approximate solutions from which stress intensity factors are extracted. In this section, a brief description of the method is presented. Additional details can be found in $[2,6,9,14]$ and the references therein.

Consider a mesh of linear finite elements in $\mathbb{R}^{n}, n=1,2$ or 3 . Let $\mathbf{x}_{\alpha}$ denote a vertex node in this mesh. The union of the finite elements sharing node $\mathbf{x}_{\alpha}$ is denoted by $\omega_{\alpha}$ and it corresponds to the support of the global finite element shape function $\varphi_{\alpha}$ associated with that node. Let $N$ denote the number of nodes and shape functions in a finite element mesh. The functions $\varphi_{\alpha}, \alpha=1, \ldots, N$, have the following property

$$
\sum_{\alpha} \varphi_{\alpha}(\mathbf{x})=1 \quad \forall \mathbf{x} \in \Omega,
$$

i.e., the finite element shape functions constitute a partition of unity. The generalized finite element method explores this property to build shape functions as described below. The GFEM is mathematically equivalent to other methods like the $h p$ Cloud method $[3,4]$ and the extended finite element method $[7,15]$.

Let $\chi_{\alpha}\left(\omega_{\alpha}\right)=\operatorname{span}\left\{L_{i \alpha}\right\}_{i \in \mathcal{I}(\alpha)}$ denote local spaces defined on $\omega_{\alpha}, \alpha=1, \ldots, N$, where $\mathcal{I}(\alpha), \alpha=$ $1, \ldots, N$, are index sets and $L_{i \alpha}$ denotes local enrichment functions.

Suppose that the finite element shape functions, $\varphi_{\alpha}$, are linear functions and that

$$
\mathcal{P}_{p-1}\left(\omega_{\alpha}\right) \subset \chi_{\alpha}\left(\omega_{\alpha}\right), \alpha=1, \ldots, N,
$$

where $\mathcal{P}_{p-1}$ denotes the space of polynomials of degree less or equal to $p-1$. The generalized finite element shape functions of degree $p$ are defined by $[2,6,9,13,14]$

$$
\left.\mathcal{F}_{N}^{p}=\left\{\phi_{i}^{\alpha}=\varphi_{\alpha} L_{i \alpha}, \alpha=1, \ldots, N, i \in \mathcal{I}(\alpha) \text { (no sum on } \alpha\right)\right\}
$$

There is considerable freedom in the choice of the local spaces $\chi_{\alpha}$. The most obvious choice for a basis of $\chi_{\alpha}$ is polynomial functions which can approximate smooth functions well. In this case, the GFEM is essentially identical to the classical FEM. The enrichment functions can also be non polynomial like those functions given by Eq. (3). This type of enrichment is used in the example of Section 4 and it is very effective to model the behavior of the elasticity solution in the neighborhood 
of cracks. Additional details on the construction and implementation of this type of enrichment can be found in, e.g., [2].

The GFEM shape functions defined in Eq. (13) are used to construct finite dimensional spaces used in the Galerkin method, like in the standard finite element method. Details can be found in, e.g., $[2]$.

\section{Center-Cracked Panel with Partially Loaded Crack Faces}

In this section, a numerical example of a finite center-cracked panel with partially loaded crack faces is presented. The geometry, boundary conditions and mesh used are illustrated in the Figure 2.

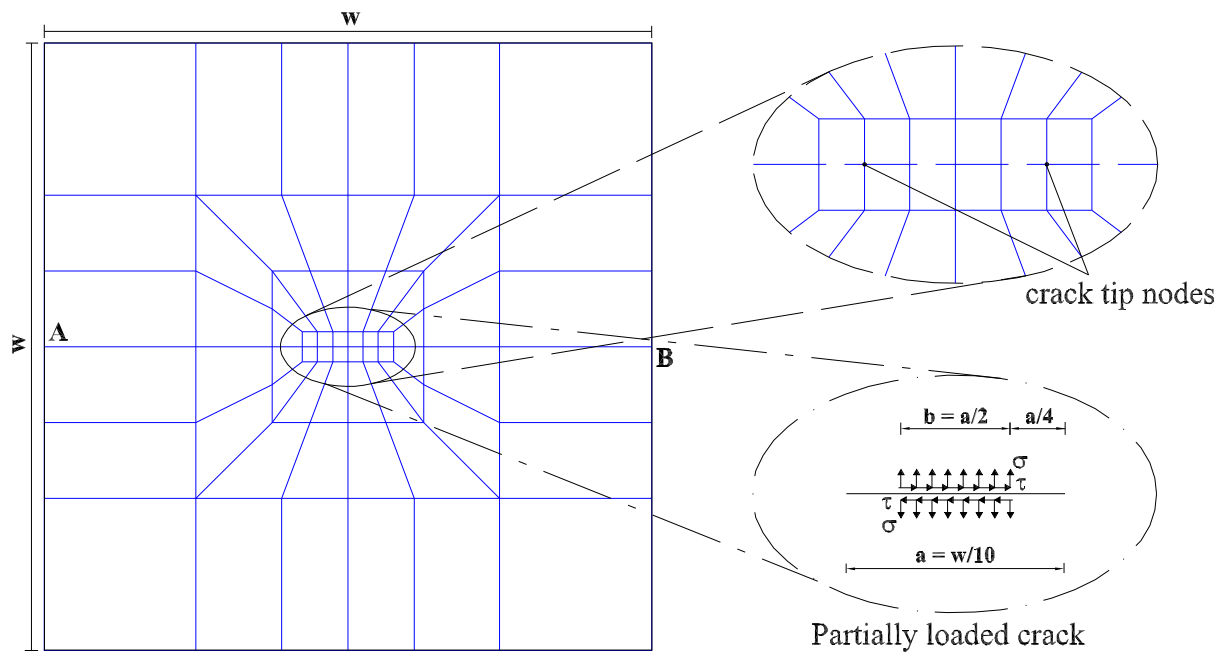

Figure 2: Domain of analysis and mesh design.

A state of plane stress is assumed and the following parameters are adopted: $w=1.0, a=w / 10$, $b=a / 2$, Poisson's ratio $\nu=0.3$, Young's Modulus $E=1.0$, and unit thickness. Point Dirichlet boundary conditions $u_{x_{A}}=u_{y_{A}}=0.0$ and $u_{y_{B}}=0.0$ at points $A$ and $B$, respectively, are set in order to prevent rigid body motion. The tractions applied at the top, $\mathbf{p}^{3}$, and lower, $\mathbf{p}^{4}$, crack faces are taken as (Cf. Fig. 2)

$$
\begin{aligned}
& \mathbf{p}^{3}=-\sigma \mathbf{n}-\tau \mathbf{t} \\
& \mathbf{p}^{4}=-\sigma \mathbf{n}+\tau \mathbf{t}
\end{aligned}
$$

where $\mathbf{n}$ is the normal vector to the faces and $\mathbf{t}$ the tangent vector pointing away from a crack tip. In the computations, $\sigma=\tau=1.0$ is adopted.

The $p$ version of the GFEM is used to solve this problem. The nodes at the crack tips are enriched with polynomial and the singular Westergaard functions given in (3). All other nodes are enriched only with polynomial functions. The mesh used is quite coarse to resolve the singularity at the crack tip using the standard finite element method. However, the use of singular enrichment functions at the crack tip is very effective. Details on the $p$ version of the GFEM can be found, for example, in [2,9].

The numerical solutions are computed using the computer program "Illinois Scientific and Engineering Toolkit (ISET)". This software is an object oriented implementation of the generalized finite 
element method. The numerical integrations for the CIM is performed using 12 Gauss points for the circular domain, $\Gamma_{2}$. The integration along the crack faces is done only over the part of the face with non-zero prescribed traction.

The example described here is a mixed mode problem. In order to validate the solution obtained from the CIM implementation, the following reference values are used:

$$
\left\{\begin{array}{l}
K_{I}^{\mathrm{inf}} \\
K_{I I}^{\mathrm{inf}}
\end{array}\right\}=\frac{2}{\pi}\left\{\begin{array}{l}
\sigma \\
\tau
\end{array}\right\} \sqrt{\pi \frac{a}{2}} \sin ^{-1} \frac{b}{a}=0.13211091\left\{\begin{array}{l}
\sigma \\
\tau
\end{array}\right\}
$$

These reference values hold for an infinite domain and they can be found in [18]. In the present case, the computations are done on a finite domain. Therefore, due to the domain finiteness, it is expected that the computed stress intensity factors are larger than those for an infinite domain. A detailed discussion on the effect of finite domain size can be found in [1].

Figure 3(a) shows the computed stress intensity factors versus the number of degrees of freedom. The polynomial order of the enrichment functions ranges from $p=1$ to $p=7$. Singular enrichment functions are used at crack tip nodes as discussed above. In the computations, the radius of the extraction domain for the CIM, $\rho_{2}$, is taken as 0.040. Figure 3(a) and Table 1 show that the values are in good agreement with the reference values for a infinite domain. The stress intensity factor $K_{I}$ $\left(K_{I I}\right)$ converges to a value about $2.5 \%(2.0 \%)$ larger than the reference value for an infinite domain. Table 1 also shows the convergence of the energy release rate with the enrichment of the solution. The energy release rate is computed using the following relation

$$
\mathcal{G}=\left(K_{I}^{2}+K_{I I}^{2}\right) \frac{1+\kappa}{8 G}
$$

The values computed for energy release rate are also in good agreement with the reference values. The energy release rate converges to a value about $4.5 \%$ larger than the reference value.

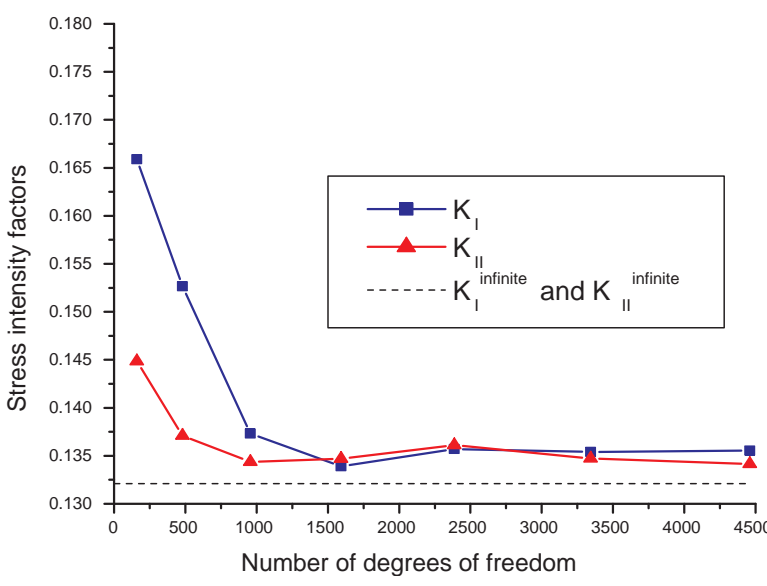

(a) Convergence of stress intensity factors.

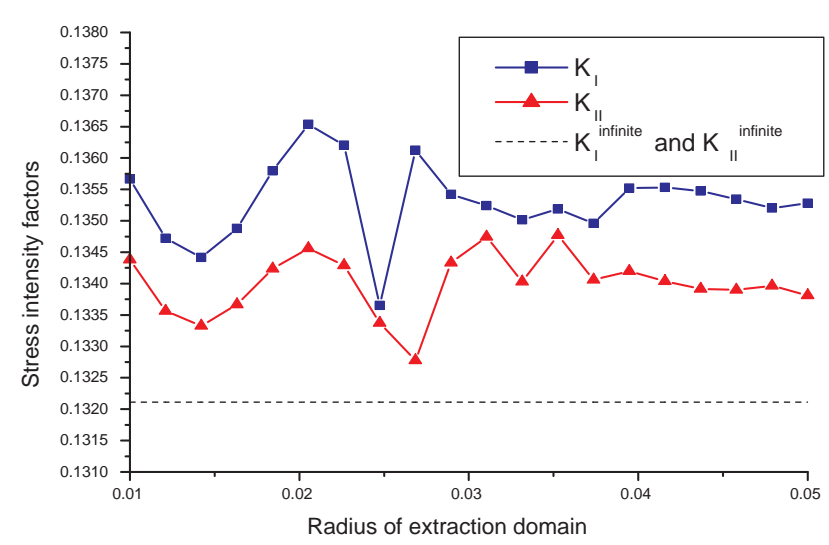

(b) Numerical verification of path independence.

Figure 3: Convergence of extracted quantities and numerical verification of path independence.

Figure 3(b) and Table 2 present the numerical verification of the path independence for the CIM. In the computations, polynomial enrichment of degree $p=6$ and singular enrichment functions at crack tip nodes are used. The results show that the CIM for loaded cracks is quite robust to variations in the dimension of the extraction domain. The values presented on Table 2 confirm that the variation on the stress intensity factors values with respect to the radius is not significant. All the values computed are very close to the average. 
Table 1: Relative error, $e_{r}\left(K_{I}\right)=\left|K_{I}^{\mathrm{inf}}-K_{I}\right| / K_{I}^{\mathrm{inf}} * 100$, for stress intensity factors and energy release rate

\begin{tabular}{ccccc}
\hline$p$ & $N$ & $e_{r}\left(K_{I}\right) \%$ & $e_{r}\left(K_{I I}\right) \%$ & $e_{r}(\mathcal{G}) \%$ \\
\hline 1 & 160 & 25.57479 & 9.650293 & 38.96117 \\
2 & 480 & 15.55442 & 3.7666 & 20.6016 \\
3 & 956 & 3.941453 & 1.717564 & 5.751966 \\
4 & 1592 & 1.356504 & 1.959785 & 3.344684 \\
5 & 2388 & 2.728836 & 3.023286 & 5.834758 \\
6 & 3344 & 2.496455 & 1.968868 & 4.516096 \\
7 & 4460 & 2.586531 & 1.551038 & 4.182635 \\
\hline
\end{tabular}

It can be observed in Figure 3(b) that there is a larger variation on the computed stress intensity factors for extraction domains with radius $\rho_{2}$ of about 0.025 than for other values. This variation is caused by the discontinuity of the tractions applied at the crack faces. However, the overall stability of the CIM is not affected (Cf. Table 2).

Table 2: Average and standard deviation for stress intensity factors in the numerical verification of the path independence.

\begin{tabular}{lcc} 
& $K_{I}$ & $K_{I I}$ \\
\hline Average & 0.135310 & 0.134007 \\
Standard deviation & 0.000650 & 0.000492 \\
\hline
\end{tabular}

\section{$5 \quad$ Summary and Conclusions}

A generalization of the contour integral method for cracks with tractions applied to their faces is presented. The formulation can handle single- as well as mixed-mode cracks. Based on the numerical experiments presented here and in $[10,11]$ the following conclusions can be drawn. The contour integral method is very robust. The computed SIFs have shown to be quite insensitive to the dimensions of the extraction domains. This is in contrast with methods based on the J-integral which require that the extraction domains to be sufficiently close to the crack tips. This limitation poses conflicting requirements on the size of the extraction domains that can be used with these methods since numerical solutions are in general of lesser quality near a crack tip. The CIM, on the other hand, allows the use of large extraction domains.

The results presented in Section 4 indicate that it is possible to compute quite accurate stress intensity factors using the CIM for loaded cracks. The results also show that a coarse mesh enriched with singular functions at the crack tip nodes is very effective for the computation of stress intensity factors.

Acknowledgments: The partial support of this work by The University of Illinois at UrbanaChampaign is gratefully acknowledged. The authors also wish to thank Prof. Ivo Babuška from the University of Texas at Austin for fruitful discussions during the course of this research. 


\section{References}

[1] David Broek. Elementary Engineering Fracture Mechanics. Martinus Nijhoff Publishers, Netherlands, fourth edition, 1987.

[2] C. A. Duarte, I. Babuška, and J. T. Oden. Generalized finite element methods for three dimensional structural mechanics problems. Computers and Structures, 77:215-232, 2000.

[3] C. A. M. Duarte and J. T. Oden. An $h p$ adaptive method using clouds. Computer Methods in Applied Mechanics and Engineering, 139:237-262, 1996.

[4] C. A. M. Duarte and J. T. Oden. Hp clouds - an hp meshless method. Numerical Methods for Partial Differential Equations, 12:673-705, 1996.

[5] F. Z. Li, C. F. Shih, and A. Needleman. A comparison of methods for calculating energy release rates. Engineering Fracture Mechanics, 21(2):405-421, 1985.

[6] J. M. Melenk and I. Babuška. The partition of unity finite element method: Basic theory and applications. Computer Methods in Applied Mechanics and Engineering, 139:289-314, 1996.

[7] N. Moes, J. Dolbow, and T. Belytschko. A finite element method for crack growth without remeshing. International Journal for Numerical Methods in Engineering, 46:131-150, 1999.

[8] B. Moran and C. F. Shih. A general treatment of crack tip contour integrals. Int. J. Fract., 305:295-310, 1987.

[9] J. T. Oden, C. A. Duarte, and O. C. Zienkiewicz. A new cloud-based $h p$ finite element method. Computer Methods in Applied Mechanics and Engineering, 153:117-126, 1998.

[10] J. P. Pereira and C. A. Duarte. Computation of stress intensity factors for pressurized cracks using the generalized finite element method and superconvergent extraction techniques. In $X X V$ Iberian Latin-American Congress on Computational Methods in Engineering, Recife, Brazil, Oct. 2004.

[11] J. P. Pereira and C.A. Duarte. Extraction of stress intensity factors from generalized finite element solutions. Submitted for publication in Engineering Analysis with Boundary Elements, 2004.

[12] J. R. Rice. A path independent integral and the approximate analysis of strain concentration by notches and cracks. Journal of Applied Mechanics, 35, 1968.

[13] T. Strouboulis, I. Babuška, and K. Copps. The design and analysis of the generalized finite element mehtod. Computer Methods in Applied Mechanics and Engineering, 81(1-3):43-69, 2000.

[14] T. Strouboulis, K. Copps, and Babuška I. The generalized finite element method. Computer Methods in Applied Mechanics and Engineering, 190:4081-4193, 2001.

[15] N. Sukumar, N. Moes, B. Moran, and T. Belytschko. Extended finite element method for three-dimensional crack modelling. International Journal for Numerical Methods in Engineering, 48(11):1549-1570, 2000.

[16] B. A. Szabo and I. Babuška. Computation of the amplitude of stress singular terms for cracks and reentrant corners. In T. A. Cruse, editor, Fracture Mechanics: Nineteenth Symposium, ASTM STP 969, pages 101-124, 1988. 
[17] Barna Szabo and Ivo Babuška. Finite Element Analysis. John Wiley and Sons, New York, 1991.

[18] H. Tada, P. Paris, and G. Irwin. The Stress Analysis of Cracks Handbook. ASME Press, New York, 3rd edition, 2000. 